\title{
Distal radioulnar joint instability with three different injury patterns assessed by three-dimensional C-arm scans: a cadaveric study
}

Journal of Hand Surgery

(European Volume)

2019, Vol. 44(10) 1072-1078

(C) The Author(s) 2019

Article reuse guidelines:

sagepub.com/journals-permissions

DOI: $10.1177 / 1753193419870378$

journals.sagepub.com/home/jhs

@SAGE

\author{
Benedict Swartman ${ }^{1}$, Laura Benner ${ }^{2}$, Jochen Franke ${ }^{1}$, \\ Paul A. Grützner ${ }^{1}$, Sven Y. Vetter ${ }^{1}$ and Marc Schnetzke ${ }^{1}$
}

\begin{abstract}
The aim of this study was to establish a radiographic method for detecting distal radioulnar joint instability intraoperatively. We created three successive instabilities in 12 intact Thiel-fixed upper limb specimens: lesion of the triangular fibrocartilage complex, Galeazzi injury and Essex-Lopresti injury. We made threedimensional scans of the wrists in pronation and supination with a mobile $\mathrm{C}$-arm. We used four validated measurement methods to quantify ulnar migration in the standard axial planes: the radioulnar line method, the subluxation ratio method, the epicentre method and the radioulnar ratio method. All types of instability showed significantly increased migration of the ulna compared with the control group. The subluxation ratio and the radioulnar ratio methods showed the highest sensitivity and specificity in detecting the instabilities. We conclude that these two methods are feasible for radiographically assessing distal radioulnar joint instability. Instability may be assumed if the migration of the ulna in the sigmoid notch is at least $45 \%$ of the length of the sigmoid notch, in which case wire transfixation of the distal radioulnar joint is advised.
\end{abstract}

\section{Keywords}

Distal radioulnar joint instability, Essex-Lopresti injury, Galeazzi, triangular fibrocartilage complex, intraoperative 3-D imaging

Date received: 15th March 2019; revised: 22nd July 2019; accepted: 26th July 2019

\section{Introduction}

The clinical examination of the stability of the distal radioulnar joint (DRUJ) remains challenging due to poor sensitivity, specificity and reproducibility (Pickering et al., 2016; Wijffels et al., 2012). Even in the most severe instability, rupture of the interosseous membrane of the forearm, a sensitivity of only $70 \%$ was obtained in the clinical examination of cadaveric specimens (Kachooei et al., 2015). Comparisons of radiologic and clinical examination methods revealed only weak agreement with kappa values between 0.01 and 0.12 (van Leerdam et al., 2017). Three-dimensional (3-D) image reconstruction was used for determining DRUJ instability, and four radiologic measurement methods based on axial 3-D reconstructions of the DRUJ have been described for the quantification of radioulnar translation (Mino et al., 1983, 1985). These measurements had a reliability of $80 \%-97 \%$ (Park and Kim, 2008; Wijffels et al., 2016). In a preliminary study, we were able to demonstrate the feasibility of a cone beam CT scan with a mobile $\mathrm{C}$-arm for assessing the physiological radioulnar translation in uninjured cadaveric specimens (Swartman et al., 2019). However, in that study we did not test instability caused by injury.

The aim of this study was to evaluate cone beam CT scan based measurement methods to detect DRUJ instability caused by specific injury patterns

\footnotetext{
1Department for Orthopedics and Orthopedic Trauma, Trauma Surgery Clinic at Heidelberg University, Ludwigshafen, Germany ${ }^{2}$ Institute of Medical Biometry and Informatics, University of Heidelberg, Heidelberg, Germany
}

\section{Corresponding Author:}

Marc Schnetzke, Ludwig-Guttmann-Str. 13, 67071 Ludwigshafen, Germany.

Email: Marc.schnetzkeabgu-ludwigshafen.de 
in human cadaveric specimens and to establish their sensitivity and specificity.

\section{Materials and methods}

\section{Specimens}

Twelve complete, macroscopically and radiologically intact human upper limb specimens with no indications of previous injuries were included in the study. They were provided by the anatomical department of the University of Graz, Austria. Ethical approval for the study was given by the local ethical board (Rhineland-Palatinate Local Ethics Committee, 55019 Mainz, Germany, No. 837.299.17). The specimens were unpaired and included the shoulder joint. The sex was unknown, and the mean age of the deceased was 74 years (range 68-84). The specimens were fixed according to Thiel's procedure and were therefore realistic in terms of consistence, elasticity and colour (Thiel, 1992; Völlner et al., 2017).

\section{Destabilizing procedures}

The specimens were prepared according to the technique described by Kachooei (Kachooei et al., 2015). Three types of injury were created for each specimen: a lesion of the triangular fibrocartilage complex (TFCC), a Galeazzi injury and an Essex-Lopresti injury. The preparations were performed by a single operator (B.S.). The skin was incised between the lateral epicondyle and the posterior aspect of the DRUJ. The DRUJ and TFCC were visualized in the space between the extensor carpi ulnaris tendon and the extensor digiti minimi tendon. The anterior and posterior radioulnar ligament of the TFCC were dissected, and the TFCC was detached from the ulnar head. The Galeazzi injury was simulated by partially dissecting the intraosseous membrane (IOM) in the space between the extensor carpi radialis muscle and the extensor digitorum communis muscle. Since in vivo the membrane is, by definition, ruptured distally to the radial fracture and the central portion is preserved, the membrane was sharply detached from the distal radius in the distal third of the forearm while sparing the central portion. Since stability testing of the DRUJ during operation of patients with Galeazzi fractures is not performed until after plating the distal radial fracture, osteotomy of the radius could be omitted in our set-up. The Essex-Lopresti injury model was produced by resecting the radial head while preserving the attachment of the distal biceps tendon, and the remaining IOM was sharply detached from the radius in the same window as in the preparation of the Galeazzi injury.

\section{3-D radiological measurements}

A fixation device was devised to fix the specimens with the elbows flexed at $90^{\circ}$ while allowing a controlled maximum supination and pronation. The position in rotation of the forearm was ensured with a rod that was fixed to the hand. Degrees were measured by goniometer and the averages calculated. 3-D scans of the wrist were taken at maximum pronation and maximum supination with a 3 -D-capable image intensifier (Arcadis Orbic, Siemens Healthcare $\mathrm{GmbH}$, Erlangen, Germany). This is a CT scan built in the form of a $\mathrm{C}$-arm. The system combines a motorized $\mathrm{C}$-arm with an additional computer and is therefore able to acquire and to edit 3-D data sets. In the 3-D data volume generated, the standard axial plane $90^{\circ}$ to the radial shaft axis was reconstructed based on freely selectable layers, similar to the methods of Park and Wijffels (Park and Kim, 2008; Wijffels et al., 2016). The selection of the correct layer was based on the depiction of the largest extension of the sigmoid notch, Lister's tubercle and the ulnar styloid process. Measurement of the images was performed with a ruler on paper at 1.5-fold magnification.

The following four measurement methods have already been described in our preliminary study (Swartman et al., 2019): the radioulnar line (RL) method (Mino et al., 1983, 1985), the subluxation ratio (SR) method (Park and Kim, 2008), the epicentre (EC) method (Wechsler et al., 1987) and the radioulnar ratio (RR) method (Lo et al., 2001). The measurement methods are depicted with their auxiliary lines in Figure 1 with details of distances and lengths.

All methods quantify the position of the ulnar head relative to the sigmoid notch of the distal radius and recording a length ratio in percentages. The distances vary between the measurement techniques, but the reference is always the length of the radial sigmoid notch. Similarly to the preceding studies (Park and Kim, 2008; Wijffels et al., 2016), posterior dislocations were quantified with positive values and anterior dislocations with negative values. Anterior dislocation was defined as a migration of the ulnar head in anterior direction. If the auxiliary lines extended beyond the ulnar on both sides, for example in the case of a disproportionately large ulnar head, the two values with their corresponding prefixes were added together. Thus, for each specimen in its intact condition, as well as after each destabilizing procedure, the four measurement methods were used in both pronation and supination. The 384 $(12 \times 4 \times 4 \times 2)$ measurements were taken in random sequence by a blinded investigator (B.S.). Values in pronation and supination were subtracted from each other to demonstrate the ulnar migration between both situations: $\Delta_{\mathrm{s}}=\mathrm{Pro}_{\mathrm{s}}-\mathrm{Sup}_{\mathrm{s}}$. 


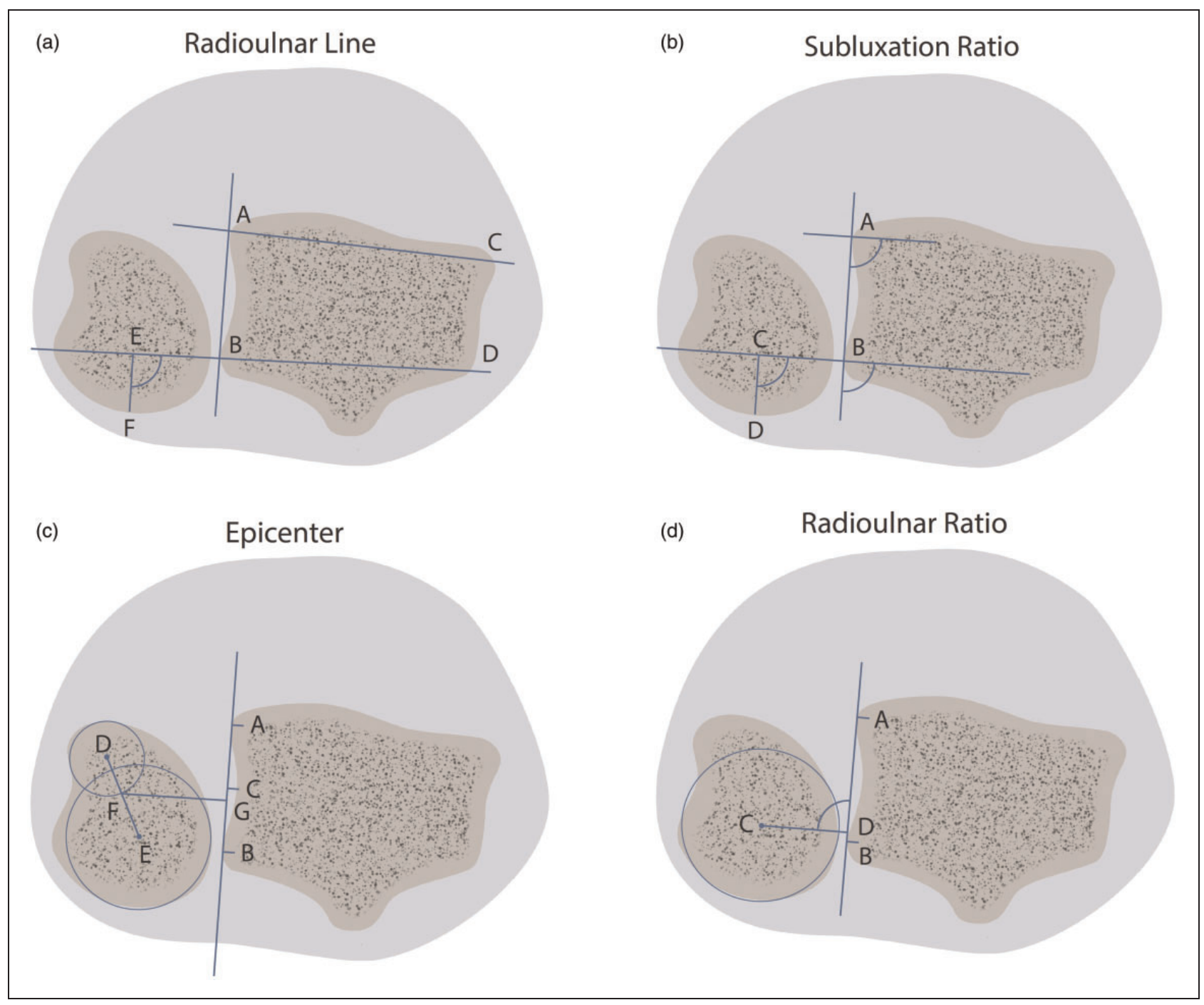

Figure 1. Methods for quantifying the position of the ulnar head relative to the sigmoid notch of the distal radius on 3-Dscans. (A) The RL method. AB marks the sigmoid notch, AC the anterior border, and BD the posterior border of the distal radius. EF marks the overlay of the ulnar head in regard to $A C$ or $B D$ and is related to the length of $A B$. (B) The $S R$ method. Line $A B$ marks the sigmoid notch, $C D$ the overlay of the ulnar head in regard to the perpendicular lines through $A$ or $B$. The length of $C D$ is related to $A B$. (C) The epicentre method. $A B$ marks the sigmoid notch, $C$ the mid-distance, $D$ is the centre of the styloid process, $E$ of the ulnar head. F marks the middle between D and $E$ and $F G$ the perpendicular line to AB. CG is related to the length of $A B$. (D) The RR method. $A B$ shows the sigmoid notch. $C D$ marks the perpendicular line to $A B$ through the centre of the ulnar head. The length of $A D$ is related to $A B$.

\section{Statistical methods}

The means of radioulnar migration distance within the control group (intact wrists) and within the different instabilities were determined, and differences were calculated using Student's $t$-test with the level of significance at 0.05 . Receiver operating characteristic curves were generated, and their areas under the curve (AUC) calculated to determine sensitivity and specificity of the measurement methods in detecting the different types of radioulnar instability. The associated sensitivities and specificities were calculated, and the associated optimal cutoff value determined using Youden's index. An area of 1 represents a perfect test; an area of 0.5 represents a worthless test.

\section{Results}

The mean maximum range of motion of the tied down uninjured limbs was $87^{\circ}$ (SD 3.4 ) pronation and $87^{\circ}$ (SD 3.7) supination. Differences were found between the means in the uninjured control group and the TFCC, Galeazzi and Essex-Lopresti groups according 
to the measurement method (Table 1). The RR method, in particular, showed significant differences $(p<0.01)$ in all injuries from the control. No significant difference was found with the RL method for EssexLopresti injuries ( $p=0.132$ ). The most clear-cut differences in radioulnar translation were observed in TFCC lesions for the RR method (TFCC 65\% vs. Control 38\%; $p=0.009$ ), in Galeazzi injuries for the RR method (Galeazzi 68\% vs. Control 38\%; $p<0.001$ ) and in Essex-Lopresti injuries for the EC (Essex-Lopresti $9 \%$ vs. Control $-10 \%$ ) and RR (Essex-lopresti 58\% vs. Control 38\%) methods (both $p<0.001$ ).

The AUCs, cutoff values, sensitivity and specificity can be found in Table 2. The RR method had the

Table 1. Ulnar head migration in the radial sigmoid notch according to four measurement methods.

\begin{tabular}{|c|c|c|c|c|}
\hline \multirow[b]{2}{*}{ Pathological condition } & \multirow[b]{2}{*}{ Measurements } & \multicolumn{2}{|c|}{$\begin{array}{l}\text { Ulnar head migration } \\
\text { (in } \% \text { of the length of the sigmoid notch) }\end{array}$} & \multirow[b]{2}{*}{$p$-value } \\
\hline & & Mean & Standard deviation & \\
\hline \multirow[t]{4}{*}{ Normal controls } & $\mathrm{RL}$ & 30 & 33 & - \\
\hline & SR & 25 & 33 & - \\
\hline & $\mathrm{EC}$ & -10 & 18 & - \\
\hline & $\mathrm{RR}$ & 38 & 16 & - \\
\hline \multirow{4}{*}{$\begin{array}{l}\text { Triangular fibrocartilage } \\
\text { complex lesion }\end{array}$} & $\mathrm{RL}$ & 62 & 43 & 0.027 \\
\hline & SR & 65 & 49 & 0.010 \\
\hline & EC & 10 & 37 & 0.048 \\
\hline & $\mathrm{RR}$ & 65 & 37 & 0.009 \\
\hline \multirow[t]{4}{*}{ Galeazzi lesion } & $\mathrm{RL}$ & 78 & 50 & 0.003 \\
\hline & SR & 78 & 53 & 0.002 \\
\hline & $\mathrm{EC}$ & 19 & 43 & 0.043 \\
\hline & $\mathrm{RR}$ & 68 & 42 & $<0.001$ \\
\hline \multirow[t]{4}{*}{ Essex-Lopresti lesion } & $\mathrm{RL}$ & 54 & 50 & 0.132 \\
\hline & SR & 58 & 46 & 0.025 \\
\hline & $\mathrm{EC}$ & 9 & 31 & $<0.001$ \\
\hline & $\mathrm{RR}$ & 58 & 32 & $<0.001$ \\
\hline
\end{tabular}

RL: radioulnar line method; SR: subluxation ratio method; EC: epicentre method; RR: radioulnar ratio method.

Table 2. Characteristics of four measurement methods related to the type of injury.

\begin{tabular}{llllll}
\hline Type of injury & Measurements & AUC & Cutoff & Sensitivity & Specificity \\
\hline Triangular fibrocartilage & $\mathrm{RL}$ & 0.75 & 40 & 0.92 & 0.63 \\
complex lesion & $\mathrm{SR}$ & 0.79 & 55 & 0.83 & 0.84 \\
& $\mathrm{EC}$ & 0.73 & -7 & 0.83 & 0.63 \\
Galeazzi fracture & $\mathrm{RR}$ & 0.80 & 49 & 0.83 & 0.79 \\
& $\mathrm{RL}$ & 0.82 & 56 & 0.83 & 0.74 \\
& $\mathrm{SR}$ & 0.84 & 47 & 0.92 & 0.79 \\
Essex-Lopresti lesion & $\mathrm{EC}$ & 0.77 & 0 & 0.83 & 0.68 \\
& $\mathrm{RR}$ & 0.83 & 45 & 0.92 & 0.79 \\
& $\mathrm{RL}$ & 0.62 & 83 & 0.33 & 0.95 \\
& $\mathrm{SR}$ & 0.70 & 58 & 0.50 & 0.84 \\
& $\mathrm{EC}$ & 0.70 & 7 & 0.58 & 0.84 \\
\hline
\end{tabular}

The optimal cutoff value was determined with Youden's index. It differentiates between stable and unstable wrists. AUC: area under the curve; RL: radioulnar line method; SR: subluxation ratio method; EC: epicentre method; RR: radioulnar ratio method. 
largest AUC in the detection of TFCC lesions $(A U C=0.80$ ), the $S R$ method in the detection of Galeazzi injuries (AUC $=0.84$ ) and the EC method in the detection of Essex-Lopresti injuries $(A \cup C=0.70)$. The corresponding cutoff values for defining instability were $49 \%$ (RR/TFCC), 47\% (SR/Galeazzi) and 7\% (EC/Essex-Lopresti). Overall, the smallest AUC values were found in the detection of EssexLopresti injuries compared with other instabilities (AUC $\leq 0.7$ ). The graphical representation in case of TFCC lesions can be seen in the receiver operating characteristic curves in Figure 2.
The highest sensitivities were determined for the $\mathrm{RL}$ method in the detection of TFCC injuries and for the SR and RR methods in the detection of Galeazzi injuries (sensitivity 0.97 in each case with specificities $>0.63)$.

\section{Discussion}

We demonstrated in a previous study that the established RL, the $S R$ and the RR methods show excellent intra- and inter-rater reliabilities in measuring radioulnar translation in uninjured cadaver limbs
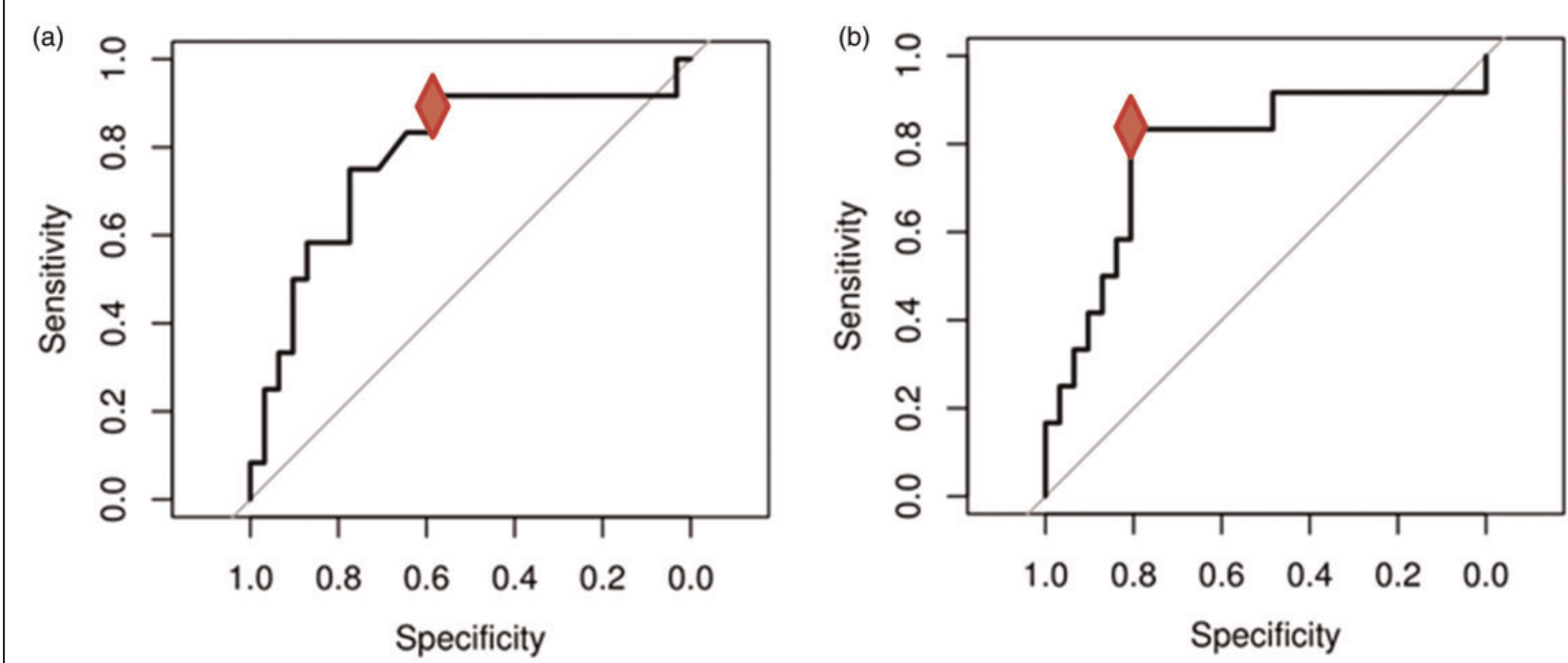

(c)

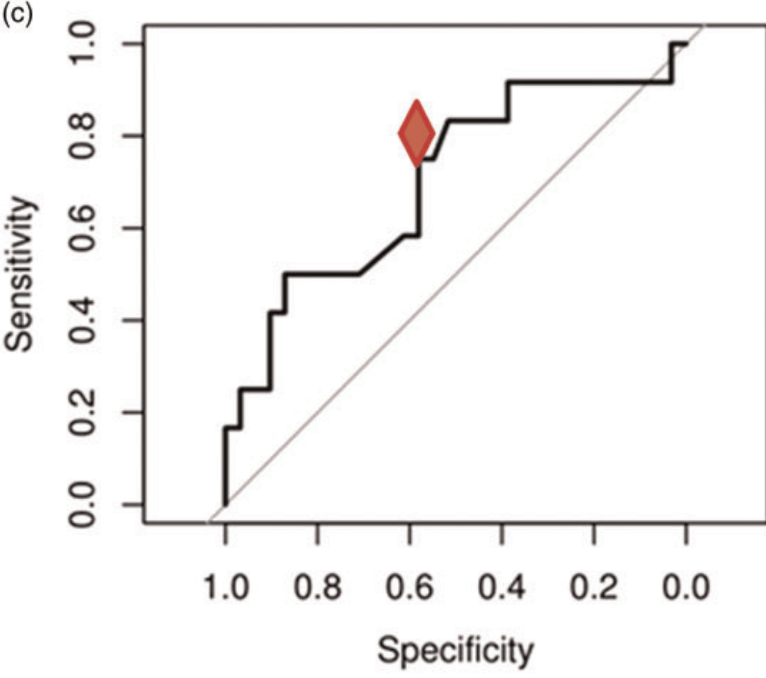

(d)

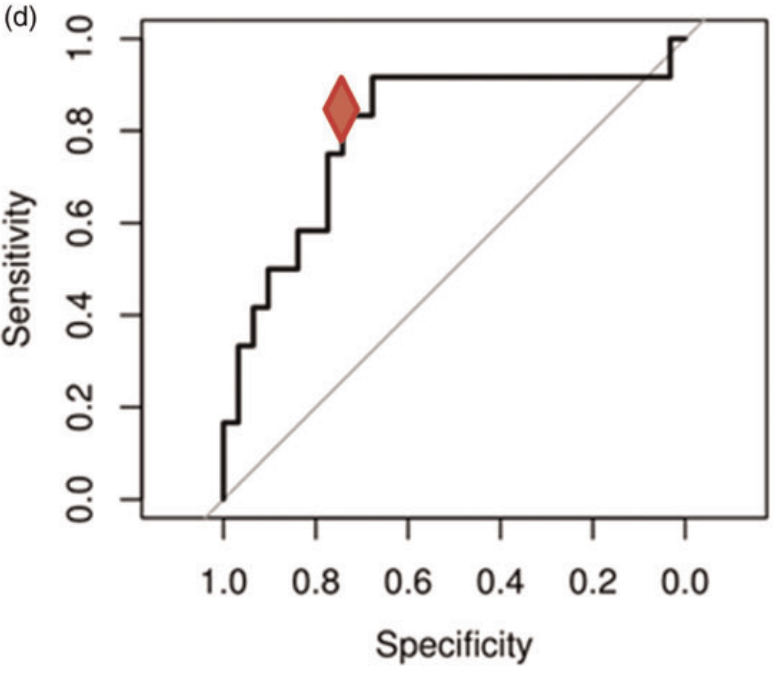

Figure 2. Receiver operating characteristic curves for each of the four measurement methods in the case of TFCC lesions. It demonstrates the relationship between sensitivity and specificity of the measurement methods. The cutoff calculated with Youden's index is marked by the diamond. The area under the curve is $\leq 1$. It represents the product of sensitivity and specificity and is a quality characteristic of each measurement method. An area of 1 represents a perfect test. An area of 0.5 represents a worthless test. (A) RL method. (B). SR method. (C) Epicentre method. (D) RR method. 
(Swartman et al., 2019). The reliability, sensitivity and specificity of these methods as diagnostic tests could now be studied systematically in three successive models of instability generated in the DRUJ of 12 specimens.

Van Leerdam et al. (2017) conducted their studies on injured wrists. They evaluated 46 patients following conservatively treated distal radial fractures. This involved examining both wrists with CT and clinical stability tests due to the suspected inter-individual variance. The authors concluded that the two examination procedures were not correlated with one another. This could mean that either the four radiological measurement methods are not reliable or that the clinical examination is unreliable. Furthermore, this demonstrates the methodological weakness, as it was not known until a later stage whether instability was present and, if so, to what extent. A very heterogeneous study population must therefore be assumed. Similarly, Wijffels et al. (2016) studied wrists following conservatively treated distal radial fractures by CT. Their study of 92 wrists revealed that the EC method is the most reliable method. However, their study was also limited by the fact that an actual instability may be suspected due to the symptoms reported by the patient but ultimately cannot be assumed with certainty.

Therefore, in our opinion, cadaveric trials are essential for the controlled investigation and establishment of an objective radiological measurement method as a diagnostic test. Kachooei et al. (2015) undertook preliminary work on the cadaveric model of different injury patterns of the forearm by simulating injuries of the TFCC and interosseous membrane in different combinations on 12 anatomical specimens, and they examined these clinically. Their results revealed a good reliability of the radius pull-push and lateral pull tests. Both tests had a sensitivity and specificity of about $70 \%$.

In our study, we intended to verify predefined injury patterns using the methods described. In comparison with the normal values, significantly different measurement results were achieved despite the high inter-individual variance. These results therefore allow a diagnosis based on a single wrist. This is feasible in the clinical setting, as the use of an intraoperative $3-\mathrm{D}$ scan with a mobile $\mathrm{C}$-arm allows immediate diagnosis.

Sensitivities and specificities of the four measurement techniques in detecting radioulnar instability were highest in the case of Galeazzi lesions. One would expect that Essex-Lopresti lesions are the most unstable and therefore most easily detectable injuries. In spite of this, the AUC is the smallest for all measurement techniques in these cases. This can be explained by the high variance of ulnar migration in each direction due to the instability in EssexLopresti injuries. The mean migration of the ulnar head lies closer to the control group than in Galeazzi or TFCC lesions.

Our study population had a mean age of 74 years with unknown sex. Transposition to the general population is therefore only possible to a limited extent. However, as the recording of normal values was undertaken on 31 specimens, we hope to have acquired results with sufficient predictive value. As the tests were performed on successively prepared limbs, the values determined are dependent variables. Therefore, the age-related tissue changes do not play an essential role in the relative changes within a specimen. A further limitation is represented by the fixed condition of the specimens. There is the possibility of distorted elasticity, particularly of the secondarily stabilized soft tissue structures. However, Völlner et al. (2017) were able to demonstrate that the ligaments of the Thiel-fixed knee were similar in terms of stiffness to those of living patients. The number of 12 specimens studied appears somewhat small for statistical evaluations. Against the background of a cadaveric study, however, these figures appear to be in line with previous publications (Kachooei et al., 2015). The demonstration of significant differences is also evidence of sufficient sample size.

From a statistical perspective, a specific measurement method cannot be recommended as the AUC, sensitivity and specificity values were very close to one another. From a practical perspective, however, the feasibility of the methods must also be taken into consideration. Intraoperative processing time and error rate must be kept to a minimum to ensure the highest possible patient safety. A simple measurement method using anatomically unequivocal landmarks and as few auxiliary lines and distances as possible is therefore essential. Furthermore, from a user's perspective, we consider sensitivity to be more relevant than specificity to minimize the chances of any DRUJ instability being overlooked. Taking these factors into consideration, we consider the RR method with measurement of the sigmoid notch and the formation of a concentric circle within the ulnar joint area to be the most suitable method.

Our findings provide the basis for using an intraoperative functional $3-D$ diagnostic procedure in patients suspected of having DRUJ instability. We had a suspicion in the case of accompanying dislocations or subluxations of the ulnar head (Fernandez Stage II), an ulnar styloid fracture near the base, that is in the proximal half (Fernandez Stage IIB), or an extension of the fracture line into the DRUJ 
(Fernandez Stage III) (Fernandez, 2001). We therefore recommend a 3-D scan of the DRUJ in both pronation and supination. For each position, the standard axial plane should be set at the level of Lister's tubercle and the ulnar styloid process, and then the RR method should be used as described above. Instability may be assumed if the ulnar migration in the sigmoid notch is greater than or equal to $45 \%$ of the length of the sigmoid notch, in which case, wire transfixation of the DRUJ is advised.

Acknowledgements Professor Friedrich Anderhuber, Macroscopic and Clinical Anatomy, Medical University of Graz, Austria, provided the anatomical specimens.

Declaration of conflicting interests The authors declared the following potential conflicts of interest with respect to the research, authorship, and/or publication of this article: Siemens Healthcare $\mathrm{GmbH}$, Erlangen, Germany, is a cooperation partner of the study group and provides ongoing financial support. No separate external financial resources were used for this specific project.

Funding The authors disclosed receipt of the following financial support for the research, authorship, and/or publication of this article: This research received funding from AOTrauma, Germany.

Ethical approval Local Ethics Committee RhinelandPalatinate, 55019 Mainz, Germany, No. 837.299.17 (11133).

\section{References}

Fernandez DL. Distal radius fracture: the rationale of a classification. Chir Main. 2001, 20: 411-25.

Kachooei AR, Rivlin M, Wu F, Faghfouri A, Eberlin KR, Ring D. Intraoperative physical examination for diagnosis of interosseous ligament rupture-cadaveric study. J Hand Surg Am. 2015, 40: 1785-90.e1.

Lo IK, MacDermid JC, Bennett JD, Bogoch E, King GJ. The radioulnar ratio: a new method of quantifying distal radioulnar joint subluxation. J Hand Surg Am. 2001, 26: 236-43.

Mino DE, Palmer AK, Levinsohn EM. The role of radiography and computerized tomography in the diagnosis of subluxation and dislocation of the distal radioulnar joint. J Hand Surg Am. 1983, 8: 23-31.

Mino DE, Palmer AK, Levinsohn EM. Radiography and computerized tomography in the diagnosis of incongruity of the distal radio-ulnar joint. A prospective study. J Hand Surg Am. 1985, 67: 247-52.

Park MJ, Kim JP. Reliability and normal values of various computed tomography methods for quantifying distal radioulnar joint translation. J Bone Joint Surg Am. 2008, 90: 145-53.

Pickering GT, Nagata H, Giddins GE. In-vivo three-dimensional measurement of distal radioulnar joint translation in normal and clinically unstable populations. J Hand Surg Eur. 2016, 41: 521-6.

Swartman B, Benner L, Grechenig S, Franke J, Grutzner PA, Schnetzke M. Normal values of distal radioulnar translation assessed by three-dimensional $\mathrm{C}$-arm scans: a cadaveric study. J Hand Surg Eur. 2019, 44: 503-9.

Thiel W. [The preservation of the whole corpse with natural color]. Annals anat. 1992, 174: 185-95.

van Leerdam RH, Wijffels MME, Reijnierse M, Stomp W, Krijnen P, Schipper IB. The value of computed tomography in detecting distal radioulnar joint instability after a distal radius fracture. J Hand Surg Eur. 2017, 42: 501-6.

Völlner F, Pilsı U, Craiovan B et al. Stability of knee ligament complex of Thiel-embalmed cadaver compared to in vivo knee. J Mech Behavior Biomed Mat. 2017, 71: 392-6.

Wechsler RJ, Wehbe MA, Rifkin MD, Edeiken J, Branch HM. Computed tomography diagnosis of distal radioulnar subluxation. Skel Radiol. 1987, 16: 1-5.

Wijffels M, Brink P, Schipper I. Clinical and non-clinical aspects of distal radioulnar joint instability. Open Orthop J. 2012, 6: 204-10.

Wijffels M, Stomp W, Krijnen P, Reijnierse M, Schipper I. Computed tomography for the detection of distal radioulnar joint instability: normal variation and reliability of four CT scoring systems in 46 patients. Skel Radiol. 2016, 45: 1487-93. 